\title{
Characteristic of Surface Partial Discharges Measured with Ultraviolet Camera
}

\begin{abstract}
P. FrąCZ, D. ZMARZEY AND T. BOCZAR
Faculty of Electrical Engineering, Automatic Control and Informatics, Opole University of Technology,

Prószkowska 76, 45-758 Opole, Poland

(Received May 12, 2014; in final form January 21, 2015)

The performed research works consider diagnosis of different models of support and bushing insulators. Insulator systems undergo aging processes, which causes deterioration of the insulating properties. In addition, pollutants that accumulate on the surface of high voltage insulators cause further reduction of the insulation properties. Presence of pollutants and high humidity level cause formation of conductive paths, which acts as a source of partial discharges. Presented results depict optical signals, recorded by ultraviolet camera, which were emitted by surface partial discharges occurring on different insulator models. In particular, the effect of the relative value of camera sensitivity on the number of surface partial discharges counts was evaluated. In addition, analysis of the effect of the surface partial discharges generation time on registered counts number was made. The experiments were performed at a constant voltage of $0.9 \mathrm{U}_{\mathrm{p}}$ and for a distance between the $\mathrm{HV}$ electrode and GND equal to $10 \mathrm{~cm}$. The experimental study lasted over a period of $30 \mathrm{~min}$, while the measurement steps were done every minute.
\end{abstract}

DOI: $10.12693 /$ APhysPolA.127.715

PACS: 42.79.Pw, 79.60.Bm, 52.80.Hc

\section{Introduction}

The subject matter of works, results of which are presented in this paper, concerns identification of opportunities and indication of application scope of optical emission phenomena accompanying partial discharges (PD) generation in high voltage insulators for diagnosis purposes. The main objective is to improve the optical spectroscopy method as applied to detection and localization of PD that may occur on bushings and support insulators. In particular, authors' aim was to identify such wavelengths of optical radiation in the range from 270 to $1700 \mathrm{~nm}$, the intensity of which would have the largest and stable value, independently of influence of various external disturbing factors. In order to achieve this goal measurement of optical radiation emitted by $\mathrm{PD}$ generated on models of support and bushing insulators were performed by using a camera, which enables recording of ultraviolet radiation.

Progressive aging of insulation components [1], which are essential for proper operation of every system for generation, transmission, and distribution of electrical energy, is an important factor for continuous and progressive growth of different methods for monitoring, analysis, and evaluation of the technical condition of electrical power devices [2-5]. Next to the electric, chemical and acoustic emission methods [6-16], the optical spectrophotometry is nowadays a widely developed technique [1726]. Optical radiation can be measured by use of a spectrophotometer. This device performs the function of a spectrometer, i.e. it generates optical spectra, and of a photometer - it takes photometric measurements of specific wavelengths of optical radiation. Optical spectroscopy can be applied only if there exists a direct line of view (called line of sight), generally in gas media and in certain cases also in liquid dielectrics. On the market there exist cameras that enable registration of optical radiation in the UV range, emitted by electrical discharges, e.g.: Lilin6, CoroCAM, CornoaScop and DayCor [20-24]. The main disadvantages of the commercial devices are: high purchase costs and relatively high power consumption (working with standard accumulators enables for operation usually up to $30 \mathrm{~min}$ ). In previous studies, the authors conducted a series of laboratory tests on systems modeling electric power insulators and have evaluated the effects of factors that may affect the obtained intensity of optical radiation emitted by PD. Among other the impact on changes in the size of intensity of recorded electromagnetic waves of the supply voltage value, during its increase and decrease process, and the impact of design parameters associated with custom-made modeling systems was estimated $[25,26]$.

\section{The scope of analysis} and the measurement procedure

In this paper results of measurements of UV radiation emitted by the PD generated on different bushings and support insulator models are presented. PD were generated on the surface of six insulators models made from porcelain: two bushing insulator models with an $4.9 \mathrm{~mm}$ inlay (IP 4.9 and IPS 4.9), two bushing insulator models, with an $9.9 \mathrm{~mm}$ inlay (IP 9.9 and IPS 9.9), two support insulator models (IWN and IWS). A detailed description of the applied devices is presented in [17] and [27]. The study aimed to determine the effect of the length of $\mathrm{PD}$ generation time on the obtained results, it was performed over a period of $30 \mathrm{~min}$, while recording data samples every minute. In addition, the impact of changes in technical parameters of the UV camera, i.e. the relative sensitivity values range from 0 to 100 , the size of the counting window (three sizes) and digital 
zoom (three values) on a number of PD counts was evaluated. Registrations were made at a constant distance between the aluminum clamps and grounded ironwork equal to $10 \mathrm{~cm}$ and for the input voltage equal to $0.9 \mathrm{U}_{\mathrm{p}}$. All measurements were performed for a fixed distance between the camera lens and the $\mathrm{PD}$ generation spot equal to $2.3 \mathrm{~m}$. A four-channel Acquitek data acquisition card type CH3160 was used, which was equipped with a SDK library. The measuring card has a resolution of 12 bit and allows for sampling rates up to $40 \mathrm{MHz}$. It has seven input ranges: $\pm 50 \mathrm{mV}, \pm 100 \mathrm{mV}, \pm 200 \mathrm{mV}$, $\pm 500 \mathrm{mV}, \pm 1 \mathrm{~V}, \pm 2 \mathrm{~V}, \pm 5 \mathrm{~V}$. During the measurements 100 and $200 \mathrm{mV}$ were applied. The input impedance is adjustable and may be selected between 1 and $50 \Omega$.

During measurements the professional camera UVollé from OFIL Systems Company was used. This camera was designed for monitoring of electric power networks of medium and high voltage. The camera is equipped with detectors recording in parallel images of PD generated on insulating systems in two bands: UV (in the range from $250 \mathrm{~nm}$ to $280 \mathrm{~nm}$ ) and the visible light. The measurement principle is based on counting the PD number detected by a matrix, which is sensitive to UV radiation (it is the number of pixels active in the UV). The count number and location of detected PD is than merged with image of the electric power object, which is obtained using the second detector: a standard CCD, sensitive to visible light. After the imposition of one image on the second one, a precise picture of the PD intensity on insulation components viewed in visible light is achieved. Within such a camera it is possible to detect, localize the occurrence, and measure the intensity of PD. Additionally, the UVollé camera is equipped with a noise reduction algorithm, by means of which sporadic and usually single UV signals are eliminated. During measurements the recorded images were displayed directly on the color LCD display, and then recorded in form of images on a SD memory card.

\section{Analyses results}

In Fig. 1 photographs of PD measured using the UV camera for exemplary chosen sample - the 15th minute of the experiment are presented. The red points depict locations of PD and their intensities. The photographs regard to the insulator models tested: (a) bushing insulator model IPS $9.9 \mathrm{~mm}$, (b) bushing insulator model IPS $4.9 \mathrm{~mm}$, (c) support insulator model IWS, (d) bushing insulator model IP $4.9 \mathrm{~mm}$, (e) bushing insulator model IP $9.9 \mathrm{~mm}$, (f) support insulator model IWN. As mentioned above the measuring procedure was performed for the same parameters of the camera, the same value of the supply voltage and the same distance between the HV and GND electrodes.

In Fig. 2 distributions of the probability density function (PDF), calculated over the number of counts, recorded during surface $\mathrm{PD}$ (SPD) generation, are presented. The particular graphs regard to the insulator models tested: (a) bushing insulator model IPS $9.9 \mathrm{~mm}$,

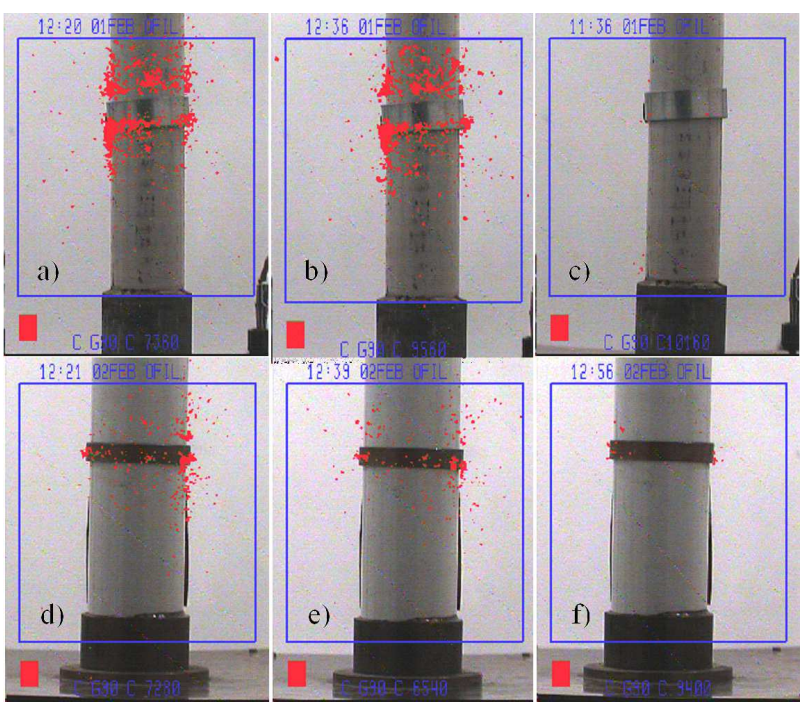

Fig. 1. Photographs of PD measured at 15 th minute of the experiment: (a) bushing insulator model IPS $9.9 \mathrm{~mm}$, (b) bushing insulator model IPS $4.9 \mathrm{~mm}$, (c) support insulator model IWS, (d) bushing insulator model IP $4.9 \mathrm{~mm}$, (e) bushing insulator model IP $9.9 \mathrm{~mm}$, (f) support insulator model IWN.

(b) bushing insulator model IPS $4.9 \mathrm{~mm}$, (c) support insulator model IWS, (d) bushing insulator model IP $4.9 \mathrm{~mm}$, (e) bushing insulator model IP $9.9 \mathrm{~mm}$, (f) support insulator model IWN. Presented characteristics were achieved for the whole 30 min long experiment while maintaining the same measurement parameters. The number of counts can be modeled using the Gauss PDF using the arithmetic mean and standard deviation of gathered intensities as parameters. The calculated curves are marked in the figure. For better analyses also the determination coefficients $R^{2}$ were calculated.

The PDF parameters and the $R^{2}$ values used for distribution model estimation for the six analyzed insulator types are listed in Table I. Higher values of the determination coefficient $R^{2}$ have been achieved for the both IPS bushing insulator models.

TABLE I

Parameters used for the PDF estimation: arithmetic mean and standard deviation of the gathered PD intensities and the determination coefficient used as fit goodness indicator.

\begin{tabular}{c|c|c|c}
\hline \hline \multirow{2}{*}{ Isolator type } & \multicolumn{3}{|c}{$\begin{array}{c}\text { Gauss PDF parameters } \\
\text { and determination coefficient }\end{array}$} \\
\cline { 2 - 4 } & $\begin{array}{c}\text { Arithmetic } \\
\text { mean }\end{array}$ & $\begin{array}{c}\text { Standard } \\
\text { deviation }\end{array}$ & $R^{2}$ \\
\hline IPS 9.9 mm & $7.6 \times 10^{3}$ & $2.72 \times 10^{3}$ & 0.759 \\
IPS 4.9 mm & $8.12 \times 10^{3}$ & $2.29 \times 10^{3}$ & 0.844 \\
IWS & $1.13 \times 10^{4}$ & $1.01 \times 10^{3}$ & 0.726 \\
IP 9.9 mm & $7.25 \times 10^{3}$ & $2.16 \times 10^{3}$ & 0.727 \\
IP 4.9 mm & $6.84 \times 10^{3}$ & $1.79 \times 10^{3}$ & 0.507 \\
IWN & $8.39 \times 10^{3}$ & $3.04 \times 10^{3}$ & 0.227
\end{tabular}


TABLE II

Comparison of the estimates determined for the model describing the dependence of the number of PD counts on the relative sensitivity and the determination coefficient $R^{2}$ obtained for the tested insulator models.

\begin{tabular}{c|c|c|c}
\hline \hline \multirow{2}{*}{ Isolator type } & \multicolumn{3}{|c}{ Model $U(A U+B)$} \\
\cline { 2 - 4 } & $A$ & $B$ & $R^{2}$ \\
\hline IPS $9.9 \mathrm{~mm}$ & -0.211 & 137 & 0.979 \\
IPS $4.9 \mathrm{~mm}$ & -0.138 & 121 & 0.919 \\
IWS & 0.805 & 43.1 & 0.961 \\
IP $9.9 \mathrm{~mm}$ & -0.362 & 128 & 0.970 \\
IP $4.9 \mathrm{~mm}$ & 0.372 & 92.8 & 0.902 \\
IWN & 1.2 & 5.63 & 0.981
\end{tabular}

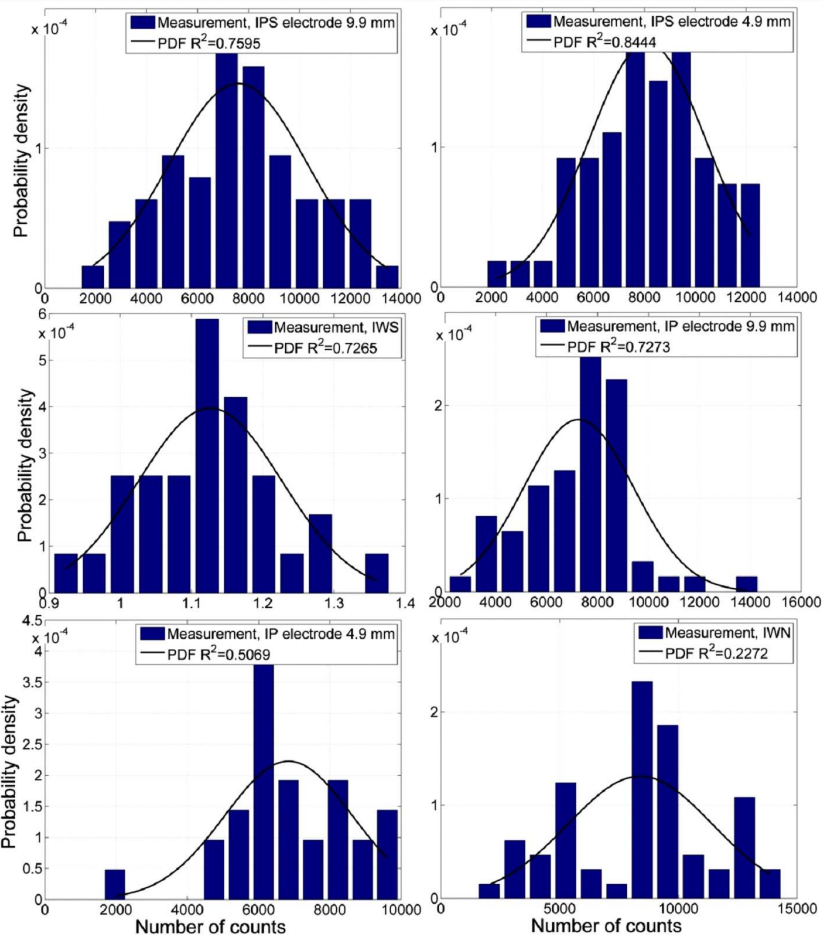

Fig. 2. Distributions of the probability density function (PDF), calculated over the number of counts, recorded during SPD generation: (a) bushing insulator model IPS $9.9 \mathrm{~mm}$, (b) bushing insulator model IPS $4.9 \mathrm{~mm}$, (c) support insulator model IWS, (d) bushing insulator model IP $4.9 \mathrm{~mm}$, (e) bushing insulator model IP $9.9 \mathrm{~mm}$, (f) support insulator model IWN.

In Fig. 3 the dependence of SPD counts on the value of the relative sensitivity, in the range from 0 to 100 is presented for the six analyzed insulator models: (a) bushing insulator model IPS $9.9 \mathrm{~mm}$, (b) bushing insulator model IPS $4.9 \mathrm{~mm}$, (c) support insulator model IWS, (d) bushing insulator model IP $4.9 \mathrm{~mm}$, (e) bushing insulator model IP $9.9 \mathrm{~mm}$, (f) support insulator model IWN. The points depict data achieved from measurements. The solid line depicts data modeled using a polynomial function: $U(A U+B)$, where $U$ is the relative sensitivity.
For better analyses of model goodness, the determination coefficients $R^{2}$ were calculated. The estimated models are characterized by a high degree of fit to the empirical data, which is indicated by a high value of $R^{2}$. For each of the considered models the $R^{2}$ was above 0.9 .

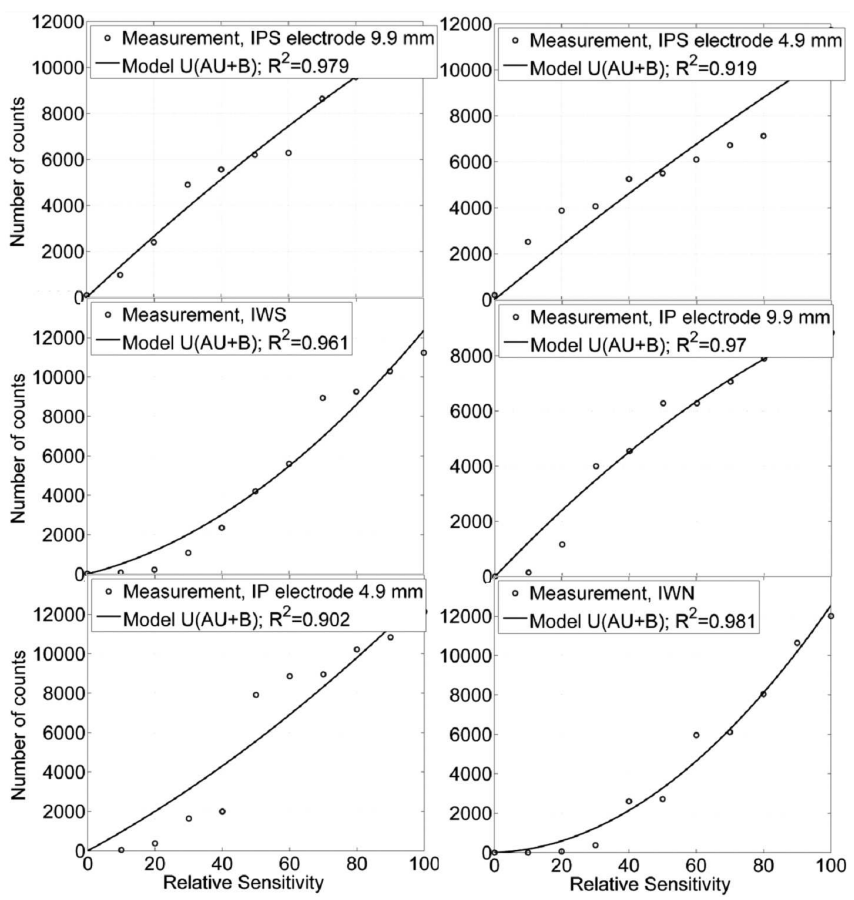

Fig. 3. Dependence of SPD counts on the value of the relative sensitivity. The points depict data achieved from measurements, while the solid line was modeled. (a) bushing insulator model IPS $9.9 \mathrm{~mm}$, (b) bushing insulator model IPS $4.9 \mathrm{~mm}$, (c) support insulator model IWS, (d) bushing insulator model IP $4.9 \mathrm{~mm}$, (e) bushing insulator model IP $9.9 \mathrm{~mm}$, (f) support insulator model IWN.

The polynomial model parameters and the $R^{2}$ values used as fit indicator estimated for the six analyzed insulator types are listed in Table II. High values of the determination coefficient $R^{2}$ have been achieved for all tested insulator models.

\section{Discussion of results and summary}

Registering the course of the $\mathrm{PD}$ generation phenomenon on the considered insulator models over $30 \mathrm{~min}$, while keeping the supply voltage at $0.9 \mathrm{U}_{\mathrm{P}}$, the other measurement parameters at constant values, as described above, a change in the counted number of PD occurs. The achieved dependence can be described by a normal Gauss distribution. Calculated values of determination coefficient $R^{2}$ were higher for the both IPS bushing insulator models.

PD counts depend on relative value of the sensitivity of the UV camera, which can be set by the user (adjustable from 0 to 100 , with gradations of 10). At the 
same time, for each of the tested insulator models, obtained intensities can be approximated using the equation: $U *(A U+B)$. Estimated models are characterized by a high degree of adaptation to the characteristics; calculated for each of the six insulator types determination coefficient takes values greater than 0.9.

In addition, the study found that the user can select one of three sizes of the window counting, the choice of which directly determines the number of PD counts. Therefore, by performing comparative measurements one size that covers the entire test area of the SPD generation should be used. Unfortunately, by such types of UV cameras, as applied in this study, it is not possible to set any desired size of the counting window size, which might cause some metrological problems during specific diagnostic measurements. Moreover, the choice of one of the four values of the digital zoom does not affect the number of counted events, related to the issue of UV radiation generated by the $\mathrm{PD}$ for each of the analyzed insulators, assuming the same window size and relative sensitivity of the camera.

On the basis of achieved results, it was found that the dominant radiation intensity occurs at intervals of approximately (300-430) $\mathrm{nm}$ and about (500-720) $\mathrm{nm}$. It was shown that the ultraviolet radiation intensity, especially UV-A, i.e. from $315 \mathrm{~nm}$ to $400 \mathrm{~nm}$, is much greater than in the range of visible radiation (VIS), i.e. (380-780) nm. These relationships were confirmed by the measurements made using a photographic technique [25] (colors: blue from about 420 to about $490 \mathrm{~nm}$, green about $550 \mathrm{~nm}$ and red from approximately 630 to approximately $780 \mathrm{~nm}$ ).

The UVolle camera from OFIL Company is an effective measurement tool to enable detection, registration and localization of PD on diagnosed insulators. The number of PD counts is displayed on the camera matrix but it does not correspond directly to the number of displayed points that represent generation of $\mathrm{PD}$, as shown in the image of the object. This may cause some difficulties in the proper interpretation of the phenomena associated with $\mathrm{PD}$, primarily the quantitative one. It should also be noted that the recorded number of PD counts is strongly dependent on the parameter settings of the camera itself. This concerns mainly the sensitivity and the counting window size. The applied camera makes it impossible to record registered events continuously (e.g., in the form of movies) and thus the subsequent evaluation in order to determine the intensity of the current UV radiation changes as a function of time. There exists only the possibility of capturing photos every few seconds which is a longer period as compared to professional cameras.

\section{Acknowledgments}

The work was co-financed from funds of the National Science Centre (NCS) as part of the OPUS programme, project no. 2013/09/B/ST8/01736.

\section{References}

[1] T. Kikuchi, S. Nishimura, M. Nagano, K. Izumi, Y. Kubota, M. Sakata, IEEE Trans. DEI 6, 548 (1999).

[2] B. Pinnangudi, R. Gorur, A. Kroese, IEEE Trans. DEI 12, 513 (2005).

[3] J. Baricos, J. Dupuy, R. Peyrous, G. Schreiber, J. Phys. D Appl. Phys. 11, L187 (1978).

[4] P. Morshuis, E. Gulski, in: IEEE Annual Report Conf. on EI and Dielectric Phenomena, IEEE, 1995, p. 327.

[5] Y. Cheng, C. Li, X. Huang, IEEE Trans. Power Delivery 23, 945 (2008).

[6] M. Szmechta, T. Boczar, P. Fracz, Acta Phys. Pol. A 120, 744 (2011).

[7] A. Cichoń, P. Frącz, D. Zmarzły, Acta Phys. Pol. A 120, 585 (2011).

[8] D. Wotzka, T. Boczar, D. Zmarzły, Acta Phys. Pol. A 116, 428 (2009).

[9] T. Boczar, D. Zmarzły, Mater. Evaluat. 62, 935 (2004).

[10] S. Borucki, T. Boczar, A. Cichoń, Arch. Acoust. 32 Supplement, 291 (2007).

[11] S. Borucki, IEEE Trans. Power Delivery 27, 670 (2012).

[12] D. Wotzka, T. Boczar, P. Frącz, Acta Phys. Pol. A 120, 767 (2011).

[13] D. Wotzka, A. Cichoń, T. Boczar, Arch. Acoust. 37, 19 (2012).

[14] P. Aksamit, D. Zmarzły, J. Electrostat. 69, 195 (2011).

[15] S. Borucki, A. Cichoń, Przeglad Elektrotechniczny 86 45 (2010) (in Polish).

[16] A. Cichoń, S. Borucki, D. Wotzka, M. Szmajda, Acta Phys. Pol. A 122, 804 (2012).

[17] P. Frącz, Acta Phys. Pol. A 120, 604 (2011).

[18] Z. Chen, P. Wang, B. Yu, in: IEEE World Automation Congress, IEEE, 2008, p. 1.

[19] I.A.D. Giriantari, in: IEEE Int. Conf. on Condition Monitoring and Diagnosis, 2008, p. 392.

[20] M. Lindner, S. Elstein, P. Lindner, J. Topaz, A. Phillips, in: 11th Int. Symp. on HV Engineering, Vol. 4, IEE, 1999, p. 349.

[21] B. Ma, W. Zhou, T. Wang, Y. Ding, in: IEEE 4th Asia-Pacific Conf. on Environmental Electromagnetics, IEEE, 2006, p. 253.

[22] N. Serdyn, D. Toit, M.Sc. Thesis, Stellenbosch University, RPA 2007.

[23] Zang Chunyan, Ye Huisheng, Lei Hongcai, Yin Xiaogen, He Junjia, Jiang Zhenglong, He Shuang, Zhao Xinjie, in: Annual Report Conf. on EI and Dielectric Phenomena, IEEE, 2009, p. 26.

[24] W. Zhou, H. Li, X. Yi, J. Tu, J. Yu, IEEE Trans. DEI 18, 232 (2011).

[25] P. Frącz, IEEE Trans. DEI 20, 1909 (2013).

[26] P. Frącz, T. Boczar, S. Borucki, A. Cichoń, D. Zmarzly, Acta Phys. Pol. A 122, 814 (2012).

[27] P. Fracz, T. Boczar, D. Zmarzły, T. Szczyrba, Acta Phys. Pol. A 124, 413 (2013), DOI. 